\title{
The Bioscience Nuclear Microscopy Program at LLNL
}

\author{
G. Bench, S. Freeman, M. Roberts, and E. Sideras-Haddad
}

This paper was prepared for submittal to the

5th International Conference on Nuclear Microprobe Technology and Applications

November 10-14, 1996

Santa Fe, NM

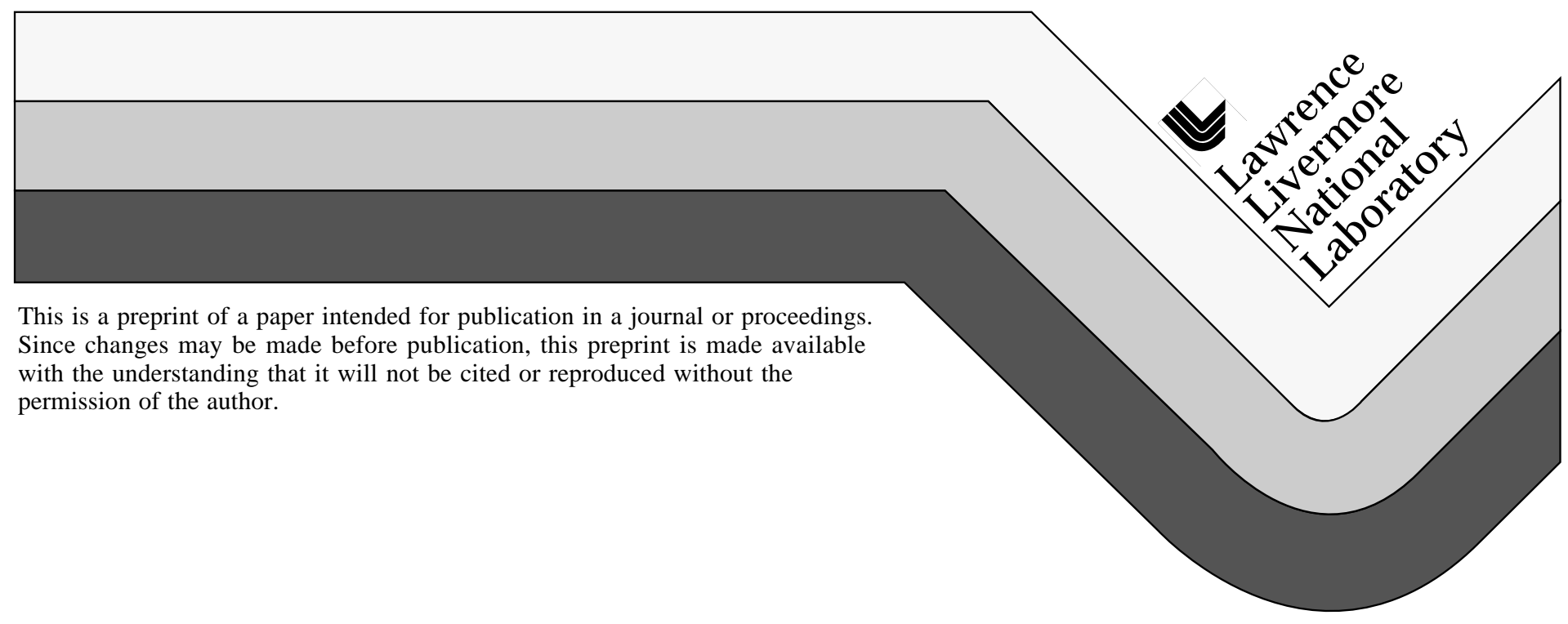




\section{DISCLAIMER}

This document was prepared as an account of work sponsored by an agency of the United States Government. Neither the United States Government nor the University of California nor any of their employees, makes any warranty, express or implied, or assumes any legal liability or responsibility for the accuracy, completeness, or usefulness of any information, apparatus, product, or process

disclosed, or represents that its use would not infringe privately owned rights. Reference herein to any specific commercial product, process, or service by trade name, trademark, manufacturer, or otherwise, does not necessarily constitute or imply its endorsement, recommendation, or favoring by the United States Government or the University of California. The views and opinions of authors expressed herein do not necessarily state or reflect those of the United States Government or the University of California, and shall not be used for advertising or product endorsement purposes. 
Reference number: 51

\title{
THE BIOSCIENCE NUCLEAR MICROSCOPY PROGRAM AT LLNL
}

\author{
G. Bench, S. Freeman, M. Roberts and E. Sideras-Haddad \\ Center for Accelerator Mass Spectrometry, \\ Lawrence Livermore National Laboratory, Livermore CA 94550
}

\begin{abstract}
Since initiation in mid 1994, a bioscience nuclear microscopy program at Livermore has enabled collaboration with bio-scientists on a variety of projects requiring quantitative elemental microanalysis. For microprobe analysis a combination of PIXE and STIM are typically used; respectively generating element distribution maps with micron scale spatial resolution, and projected densities and histological information with sub-micron spatial resolution. Current studies demonstrate the applicability of nuclear microscopy (particularly when combined with other analysis techniques) in environmental tracing, toxicology, carcinogenesis, and structural biology. The program currently uses $\sim 10$ percent of the available time on a $10 \mathrm{MV}$ tandem accelerator that is also applied to a variety of Accelerator Mass Spectrometry and other microprobe programs. The completion of a dedicated nuclear microprobe system, using a 5 SDH NEC 1.7 MV tandem accelerator and employing several energy dispersive x-ray detectors to improve x-ray counting rates, promises increased accelerator access, greater sample throughput and continued expansion of the program.
\end{abstract}

Corresponding author: Graham Bench,

Center for Accelerator Mass Spectrometry, L-397

Lawrence Livermore National Laboratory,

Livermore, CA 94550, U.S.A.

Phone: (510) 423-5155, Fax: (510) 423-7884

Email: bench1@1lnl.gov 


\section{Introduction}

The use of focused, $\mathrm{MeV}$ energy ion beams for materials characterization at the multi-user tandem laboratory (MTL) in Livermore commenced in 1987. The original microprobe was constructed to perform low current, transmission energy loss measurements requiring a spatial resolution of a few $\mu \mathrm{m}$ [1]. However, for beam currents of $100 \mathrm{pA}$, spot sizes of only $\sim 25 \mu \mathrm{m}$ could be obtained. To supplement our capabilities a milli/microprobe beamline was built in late 1991 [2] to investigate the use of Particle Induced X-ray Emission (PIXE) for non-proliferation, materials science, environmental and bio-medical applications. This system could perform PIXE analyses at $100 \mathrm{pA}$ beam currents with a spatial resolution of $10 \mu \mathrm{m}$ [2]. However, it became clear that a greater scope of projects could be addressed if PIXE analyses could be performed time efficiently at micron scale spatial resolution. Consequently, a third beamline (replacing the original microprobe) was constructed and completed in mid 1994 [3]. This later system can focus $100 \mathrm{pA}$ of protons into a spot size of $\sim 1 \mu \mathrm{m}$ and can perform low current analyses at sub-micron scale (down to $\sim 0.3 \mu \mathrm{m}$ ) spatial resolution [3]. Major programs on this microprobe include nonproliferation, materials characterization and bioscience.

\section{Bioscience Nuclear microscopy at LLNL}

The mission of the bioscience nuclear microprobe program is to provide quantitative cytometric and tissue analysis to investigators/collaborators within Lawrence Livermore National Laboratory, the University of California system and other institutions. We emphasize the term 'quantitative' as there are other micro-analysis techniques (such as secondary ion mass spectrometry or fluorescent dye staining techniques coupled with light and electron microscopy) that possess either better spatial resolution, better sensitivity or quicker/cheaper analyses. However, such techniques have difficulty in providing accurate quantitation which in many instances limits the degree of understanding obtained from the analysis. Conversely, nuclear microscopy has the unique advantage that it can measure elemental profiles within cells quantitatively at sensitivities approaching the part per million ( $\mathrm{ppm}$ ) level. Consequently, we strongly focus on establishing collaborations that need accurate elemental quantitation at sensitivities down to a few ppm. We will suggest another analysis technique if it is better suited to study of a particular problem.

It is our goal to establish close ties with all collaborators that have need of quantitative elemental micro-analysis at the cellular/sub-cellular level. We strive to obtain a thorough 
understanding of the science being studied and rarely perform measurements where we contribute no scientific input. Likewise, collaborators are also encouraged to understand the nature as well as the advantages and limitations of the microprobe analyses being performed. Collaborators are frequently present and play an active role during data acquisition and analysis and accordingly we are actively involved in the dosing, sacrifice of subjects and preparation of subject tissues. Through this form of partnership, successful working relationships tend to evolve which often lead to refined methodologies that increase the usefulness of the analyses/studies. Further, as a result of these partnerships, collaborators frequently elaborate the capabilities of nuclear microscopy to other investigators in their own or related fields. Such referrals have helped lead to further collaborative projects and program expansion.

The nuclear microprobe at Livermore is primarily setup as a measurement tool. We currently enjoy $\sim 10$ percent of the available time from a $10 \mathrm{MV}$ tandem accelerator that is also used for a variety of Accelerator Mass Spectrometry and other microprobe projects. We could gain from more accelerator time. Consequently, unless there is a particular need that will lead to widespread programmatic use, minimal time is spent on microprobe modifications or developing the use of new analysis techniques. For microprobe analysis of biological specimens a combination of PIXE and Scanning Transmission Ion Microscopy (STIM) are typically used; respectively generating element distribution maps with micron scale spatial resolution, and projected densities and histological information with sub-micron spatial resolution.

PIXE data are usually obtained using incident $3 \mathrm{MeV}$ proton microbeams. X-rays are currently detected with a $\mathrm{Si}(\mathrm{Li})$ detector that can subtend a solid angle of up to $150 \mathrm{msr}$ to the specimen. The detector is located at an angle of $135^{\circ}$ with respect to the incident beam. As biological specimens are transparent to the incident beam, charge is collected in a biased Faraday cup located behind the sample. X-rays are recorded in list mode along with coincident beam spatial co-ordinates arising from repeatedly scanning the beam electrostatically over the sample in a point by point raster mode. Within each pass a pre-determined constant amount of charge (up to $0.1 \mathrm{nC})$ is deposited at each beam location.

Owing to the thin nature of freeze dried cells and tissue sections PIXE quantitation is often obtained using the thin film approximation to obtain $\mu \mathrm{g} / \mathrm{cm}^{2}$ concentrations. Such concentrations can readily be converted to cellular elemental masses by multiplication with the area occupied by the cell or the scan area [4]. For thicker samples and analyses requiring ppm concentrations, target 
thicknesses are usually determined using the energy loss contrast STIM method reported by Lefevre et al. [5]. Depending on the specimen areal density either protons or heavy ion beams, such as Oxygen, are used. STIM data are collected either before or after PIXE data using Silicon surface barrier detectors that can be located immediately behind the sample. Energy losses are converted to areal densities or thicknesses assuming the sample composition is $\mathrm{C}_{5} \mathrm{H}_{9} \mathrm{O}_{2} \mathrm{~N}$ [5]. In addition STIM images have frequently been used to help identify boundaries and features of interest associated with element localizations in registered PIXE images. Ion backscattering analysis has also been employed to determine specimen projected densities when the need has arisen. The use of data reduction and PIXE spectrum fitting codes to obtain quantitative elemental concentrations on our microprobe system have been described in greater detail elsewhere [6].

For microprobe analysis, tissue sections and cells are usually mounted on nylon membranes of sub-micron thickness stretched over a circular aperture in a mylar target frame. The nylon membranes are strong, free of contaminants and optically transparent enabling both front and rear specimen viewing within the microprobe target chamber. Electron, optical and phase contrast microscopes are also available at Livermore for specimen study before and after microbeam irradiation. For collaborative studies with Livermore bio-scientists direct access to chemicals/reagents/glassware (for animal dosing, specimen preparation, freezing, staining, etc.) and a lyophilizer (for freeze drying of samples) exists on-site. Additionally, since we have gained considerable experience on the cryo-preparation of tissue sections through scientific interaction, the purchase of a dedicated cryo-microtome for the bioscience program is being contemplated.

Current bioscience studies include: 1) the measurement of DNA, protein and metal content in individual sperm for studies relating to fertility and DNA packaging; 2) cellular uptake and processing of metal containing cancer treating drugs; 3) heavy metal uptake in a yeast model for plant cells with potential application to environmental metal remediation; 4) the structure of skin including the effect of various insults on the epidermis; 5) an investigation of the factors controlling central nervous system (CNS) transport and toxicity of inhaled inorganics; and 6) cell metabolism of polyaromatichydrocarbon carcinogens. These studies indicate the utility of nuclear microscopy (particularly when coupled with other analysis techniques) in environmental tracing, toxicology, carcinogenesis, and structural biology. The ensuing discussion summarizes these studies, our experience with the use of a wavelength dispersive detector in microbeam PIXE analysis of biological tissue and the construction of a dedicated nuclear microprobe at Livermore. 


\section{DNA packaging in mammalian sperm}

During spermiogenesis in mammals the structure of spermatid chromatin is completely reorganized and the DNA is repackaged by small proteins called protamines. Two different types or families of protamine molecules, protamine 1 and protamine 2 , participate in this process. Pooled sample biochemical analyses have suggested that protamine defects in sperm correlate with male infertility [7]. However, these analyses are 'semi' or non-quantitative and do not allow the examination of sperm sub-populations nor the variation of protamine content within the cell population. Quantitative single cell analyses can be used to supply such information and complement pooled sample data. Nuclear microscopy has been used to measure the size, shape and mass of as well as elemental masses within individual mammalian sperm nuclei $[4,8,9,10]$. The Sulfur mass can be used to determine the total protamine (protamine 1 plus protamine 2) content of the sperm while the Phosphorus mass can be used to determine the DNA mass present within the sperm [4,8]. For sperm from fertile mammalian semen, nuclear microscopy data produces average protamine and DNA masses per nucleus that agree well with available data from pooled sample analyses [4,8]. Further, although the relative amount of protamine 1 and 2 can vary widely in fertile semen of different mammalian species, nuclear microscopy data suggests that the ratio of total protamine mass to DNA mass is similar for species in which little DNA remains packaged by histones [8].

It is also thought that the Zinc concentration in human semen and sperm is critical for male fertility [11]. Two groups have obtained evidence that suggests protamine 2 binds Zinc in vitro $[12,13]$. Both groups have proposed that protamine 2 may co-ordinate the metal in a fashion similar to Zinc finger proteins to facilitate or modulate DNA binding and use it to perform some essential function in vivo. Although these studies have focused on how Zinc may interact with sperm nuclear proteins, it is not known whether the metal is sufficiently abundant inside the sperm nucleus to be bound to protamine. Quantitative nuclear microscopy measurements of the Zinc and protamine content within several species of mammalian sperm suggest that Zinc is sufficiently abundant within normal, fertile mammalian sperm nuclei to be bound to protamine 2 [9].

Subtoxic doses of heavy metals, such as Cadmium and Lead, are also suspected to reduce male fertility $[14,15]$, possibly by modifying the proteins that package DNA in sperm. One hypothesis suggests that heavy metals, which bind tightly to free thiols, replace or compete with Zinc that is normally bound to cysteine residues in protamine and forms more stable metal - SH 
bonds. Such bonds may ultimately prevent proper decondensation of sperm chromatin following fertilization. To test this Zinc replacement hypothesis nuclear microscopy is being used to measure Zinc and Cadmium levels in individual sperm from mice that have been exposed to various doses and dose rates of $\mathrm{CdCl}_{2}$.

Nuclear microscopy has also been used to study the expression of the protamine 2 gene in several lines of transgenic mice. Nearly 2200 individual sperm/spermatids have been examined with PIXE for this study. A mouse protamine 2 gene construct was prepared by oligonucleotide mutagenesis and microinjected into the male pronuclei of fertilized mouse ova [10]. Mutations in the coding sequence of this construct were used to provide a method for discriminating (by endolys $\mathrm{C}$ digestion) between protamine 2 molecules synthesized by the endogenous and inserted genes, and to determine if the protamine 2 molecules containing these mutations are processed and compete for integration into mouse sperm chromatin. Seven founders were obtained. Sperm and late step spermatids produced by their progeny were analyzed for expression of the mutant protamine 2 gene. Using Endolysine $\mathrm{C}$ to selectively digest protamine 2 molecules synthesized by the endogenous protamine 2 gene and gel electrophoresis to resolve the digestion products, the studies have shown that males produced by three founders express the mutant protamine 2 gene at a significant level [10].

The protein content of individual spermatids and sperm was determined by PIXE. By comparing the Sulfur (protamine) content of spermatids from each transgenic line (offspring produced from a founder) to those obtained from normal males, additional protamine 2 present in the nuclei of transgenic males could be determined. Further PIXE was employed to analyze two different size classes of late-step spermatids for the transgenic and control lines - larger more immature spermatids and smaller more mature spermatids (spermatids in which the nuclear shaping is virtually complete). For one transgenic line expressing the mutant gene at a significant level, the results reveal that the larger, least mature spermatids of heterozygous males separate into two populations of cells - spermatids containing normal amounts of Sulfur in comparison to control large spermatids and those that contain $\sim 35$ percent more Sulfur on average (additional protamine produced by the transgene) [10]. In contrast, the nuclei of the smaller, most mature spermatids contain only one population of cells. The sulfur content of this population is similar to the smaller spermatids of control animals lacking the altered protamine 2 transgene [10]. Large immature spermatids isolated from homozygous males from the same line were also found to 
contain only a single population of spermatids [10]. However, these spermatids corresponded to the population of large spermatids with an increased Sulfur content in heterozygous animals. These data indicate that, for this line, the protamine synthesized by the transgene and its mRNA are confined to those cells that contain the gene. They further demonstrate that spermatids in this line do not retain the extra protamine, but begin losing it before nuclear shaping is complete and the sperm are ready to leave the testis, preserving the normal protamine 1 and protamine 2 stoichiometry. PIXE and biochemical analyses of sperm and spermatids from other transgenic lines also indicate that for those lines expressing the mutant gene at a significant level: 1) the mutant protamine 2 is present in the chromatin of late step spermatids; 2) excess protamine 2 is not integrated into chromatin but is lost as the spermatids mature; and 3) processing of the mutant protamine 2 occurs normally in all but one line - that with the largest number of integrated transgene copies [10].

These studies indicate that quantitative single cell analysis techniques such as nuclear microscopy may help resolve theories regarding the importance of protamines and metals to male fertility and help identify biochemical defects responsible for male infertility. Nuclear microscopy has already been used to observe defects in the sulfur content of individual sperm from infertile patients whose sperm were suspected of being protamine deficient. We are beginning to work with an In Vitro Fertilization (IVF) group to investigate the use of nuclear microscopy to identify subpopulations of biochemically normal sperm in infertile male semen. Since fertility is an average property of semen it is quite possible that small sub-populations of normal, fully functional sperm are present in many infertile semen samples. Nuclear microscopy measurements may help enable selection of sperm that at least possess biochemically normal profiles for fertilization of ova in micro-insemination IVF techniques.

\section{Cellular uptake and processing of metal containing cancer treating drugs}

Treatments for cancer often use DNA adduct forming agents like Cis-platin. These drugs have different treatment outcomes in individuals and, like most chemo-therapeutic agents, are not 100\% effective. Resistant cells also develop and limit the efficacy of these treatments. More data is needed on the uptake and distribution of these drugs to understand how the metal finds and interacts with DNA as well as how normal and tumor cells process these compounds. Drug concentrations also need to be measured to understand the metal distributions that result in DNA 
damage and cell death. Understanding these processes is critical to the rational design of drugs that effectively destroy tumors with minimum side effects. Nuclear microscopy allows direct quantitation of heavy metals, such as Platinum, within a cell and is being employed to gain a better understanding of how tissues process DNA damaging metals.

\section{Metal uptake in yeast: Bioengineering plants with application in metal remediation}

An efficient, inexpensive and ecologically safe bioremediation procedure for heavy metals would ideally consist of engineered plants that exhibit organ-specific accumulation of toxic metals. Strains of yeast have been developed as excellent models to study the metal detoxification and accumulation mechanisms in plants [16]. Nuclear microscopy is being employed to provide definitive information on the uptake, sites of assembly and subsequent storage of heavy metals within yeast cells and the roles that Calcium and nutritional conditions play in these processes. The data obtained from this work in conjunction with other studies [17] may help direct efforts towards the optimization of metal accumulation properties of plants that would be developed for phytoremediation.

\section{Structure of skin including the effect of various insults on the epidermis}

The formation of the epidermal permeability barrier requires lipid to be secreted from the uppermost keratinocytes in the epidermis. This secretion is controlled by extracellular Calcium concentrations. Conversely, a Calcium gradient is found within the epidermis that requires an intact barrier for its maintenance. To determine whether the barrier forms before or after the gradient, explant cultures of fetal rat skin have been prepared in which both epidermal permeability barrier and Calcium gradient formation were monitored in relation to gestational age. Formation of the barrier was determined by light microscopy and Lanthanum permeation studies as judged by electron microscopy, whilst formation of the Calcium gradient was determined by nuclear microscopy. For each animal subject the two studies were performed on separate but adjacent serial sections. The results of this work indicate that the Calcium gradient develops after the formation of the epidermal permeability barrier. Other techniques indicate that elevated levels of Calcium in bathing solutions inhibit barrier recovery after disruption, independent of the type of insult [18]. Such data suggest epidermal Calcium concentrations may be an important signal for barrier homoestasis. Consequently, nuclear microscopy is being employed to determine the effects 
that various barrier disruption maneuvers have on the Calcium and other ionic gradients [19] and the effects that induced changes in ion concentrations after barrier disruption have on barrier repair.

\section{Factors controlling CNS transport and toxicity of inhaled inorganics}

Nuclear microscopy is being used to locate and quantitatively measure metal concentrations in olfactory and brain tissue of rats that have been exposed by inhalation to several inorganics commonly found as environmental contaminants. The focus of the studies is to determine which inorganics enter the central nervous system (CNS) directly following inhalation, the form of the inhalant necessary for penetration of the CNS, the patterns of brain disposition of the metals and the rates of clearance. Physiological variables that enhance epithelial permeability and thereby increase CNS deposition of inhalants are also being examined. The major goal of this work is to help ensure sufficient data are available to derive clean-up levels of inorganic contaminants at environmental restoration sites protective of public health, while reducing unnecessary conservatism resulting from uncertainties in available data. This work is also seen to be pro-active since it is anticipated that the Environmental Protection Agency may include direct entry of inhalants to the CNS in setting future inhalation reference doses for metals.

\section{Cell metabolism of polyaromatichydrocarbon carcinogens}

The detection of Iron in cellular membranes may be related to the metabolic activity of the cell. When liver cells are exposed to polyaromatic hydrocarbon (PAH) carcinogens, the level of specific cytochrome P-450s, PAH metabolizing enzymes, increase up to 50 fold. Increased Iron should correlate with cytochrome P-450s as they have Iron centers. Movement of Iron from the cytoplasm to membranes may indicate toxic exposure. However, it is not known whether the proteins are synthesized de novo or are activated. In the former case Iron would move into the membrane with the new protein. In the latter case Iron would already be present in the protein and be activated by the exposure. The latter situation should not show mobilization of the Iron. Nuclear microscopy is being employed to quantitatively measure elemental profiles (specifically Iron) in liver cells from mice that have been exposed to PAH carcinogens to quantify and understand the response of PAH metabolizing enzymes. Such quantification may help lead to a better understanding of carcinogen and drug metabolism at the cellular level.

\section{Wavelength Dispersive microbeam PIXE analysis of biological specimens}


For x-ray detection the current microprobe employs a Princeton Gamma - Tech energy dispersive lithium drifted silicon detector and a wavelength dispersive spectrometer [20] WDX-600 manufactured by Microspec Corporation of Fremont, California. Wavelength dispersive spectrometry (WDS) possesses vastly better energy resolution than current energy dispersive spectrometry (EDS) systems - potentially lowering minimum detection limits for minor and trace elements. However, at Livermore, we have found the WDX-600 to be of limited use for microbeam PIXE analysis of biological tissues. The major limitations are the low detection efficiency compared to EDS (typically a factor between 50 and 200 smaller compared to the $\mathrm{Si}(\mathrm{Li})$ detector subtending a solid angle of $32 \mathrm{msr}$ to the target) and that the entire detectable $\mathrm{x}$-ray spectral range is not recorded simultaneously. With the WDX-600 it is necessary to use target currents of at least a few nanoamperes to obtain practical count rates that enable analyses to be performed within a few hours. This requirement limits our microprobes spatial resolution to several $\mu \mathrm{m}$ or greater which results in very coarse elemental profiles being obtained within a cell. Such profiles are of limited use for many of our investigations. Further, with the use of higher beam currents and doses analysis of fragile biological samples that are prone to beam damage is often precluded. The WDX-600 also possesses instrumental noise of up to $1 \mathrm{cps}$ even with no beam on target. This background can severely limit the instrument's utility for trace element detection.

\section{Construction of a dedicated standalone nuclear microprobe}

A dedicated standalone microprobe system is currently under construction at the MTL that will employ a NEC 5 SDH-2 1.7 MV tandem accelerator. The nuclear micropobe beamline on this system is similar to our present microprobe [3] but will have several notable improvements. The most noticeable development will be the incorporation of four EG\&G Ortec energy dispersive Iglet-X x-ray detectors (energy resolution $<145 \mathrm{eV}$ at $5.9 \mathrm{keV}$ ). These detectors will all be mounted at backward angles to the target. Further, they should subtend a combined solid angle of up to $1000 \mathrm{msr}$ to the target. By combining the signals from all the $\mathrm{x}$-ray detectors it is envisaged that either analysis times will be significantly reduced enabling higher sample throughput or that smaller beam currents will be used enabling finer spatial resolution to be obtained. Owing to the thin nature and composition of most biological specimens it is anticipated that $\mathrm{x}$-ray absorption factors within the specimen will not differ significantly for the different angular locations of the 
four detectors. The standalone microprobe system will also possess computer controlled beam transport and focusing using Labview ${ }^{\circledR}$ software, fully automated target positioning and rear and front specimen viewing video microscopes. Finally, for other measurement programs where some analyses cannot be performed in-vacuo, a ultra-thin $\mathrm{Si}_{3} \mathrm{~N}_{4}$ window in a removable flange that can be located over the beam entry port to the target chamber will provide an external beam capability for the new beamline. It is anticipated that bioscience will use up to 50 percent of available time on this standalone system. Such utilization promises continued expansion of the bioscience program.

\section{Conclusion}

The bioscience nuclear program at LLNL is now in its third year of operation. We have concentrated our efforts on providing quantitative cytometric analysis to a wide range of collaborative projects and have spent minimal time on system or technique development. Although advances in techniques should be encouraged, unless they are economically attractive, technically vital or lead to widespread programmatic use they will fail to attract scientific problems.

\section{Acknowledgments}

This work was performed under the auspices of the U.S. Department of Energy at Lawrence Livermore National Laboratory under contract W-7405-ENG-48. Reference herein to any specific commercial product does not necessarily constitute or imply its endorsement, recommendation, or favoring by the United States government or the University of California. The authors would like to thank Dan Morse and Dale Heikkinen for help with data collection and analysis. The authors gratefully acknowledge the collaboration of Rod Balhorn, Shelley Corzett, Ken Turteltaub, Jim Felton, Mike Malfatti, Bob Mauthe, Rajesh Mehra, Johnnye Lewis, Thea Mauro, Debbie Cumrine and Chris Cullander.

\section{References}

1) A.E. Pontau, A.J. Antolak, D.H. Morse, A.A. Ver Berkmoes, J.M. Brase, D.W. Heikkinen, H.E. Martz and I.D. Proctor, Nucl. Instr. Meth. B40/41, (1989), 641.

2) D.W. Heikkinen, G.S. Bench, A.J. Antolak, D.H. Morse and A.E. Pontau, Nucl. Instr. Meth. B77, (1993), 45 
3) M.L. Roberts, G.S. Bench, D.W. Heikkinen, D.H. Morse, P.R. Bach and A.E. Pontau, Nucl. Instr. Meth. B104, (1995), 13.

4) G.S. Bench, R. Balhorn and A.M. Friz, Nucl. Instr. and Meth. B99, (1995), 553.

5) H.W. Lefevre, R.M.S. Schofield, J.C. Overley, and J. McDonald, Scanning Microscopy, 1, (1987), 879.

6) A.J. Antolak and G.S. Bench, Nucl. Instr. and Meth. B90, (1994), 596.

7) R. Balhorn, S. Reed and N. Tanphaichitr, Experientia 4, (1988), 52.

8) G.S. Bench, A.M. Friz, M.H. Corzett, D.H. Morse and R. Balhorn, Cytometry, 23, (1996), 263.

9) G.S. Bench, A.M. Friz, M.H. Corzett, R. Oliva and R. Balhorn, Supplement to Molecular Biology of the Cell, 6, (1995), 428a

10) R. Balhorn, M.H. Corzett, M.H. Hwang, G.S. Bench, A.M. Friz, P.J. Mroz, J. Mazrimas, W.E Pence II, C. Weber and L. Thompson, Supplement to Molecular Biology of the Cell, 6, (1995), 316a

11) U. Kvist, L. Bjorndahl , G.M. Roomans and C. Lindholmer, Acta Physiol. Scand. 125, (1985), 297.

12) J.M. Gatewood, G.P. Schroth, C.W. Schmid and E.M. Bradbury (1990): J. Biol. Chem. 265, (1990), 20667.

13) F. Bianchi, R. Rousseaux-Prevost, P. Sautiere and J. Rousseaux, Biochem. Biophys. Res. Commum. 182, (1992) 530.

14) Parizek, J., J. Reprod. Fertil. 1, (1960), 294.

15) Cullen, M.R., Kayne, R.D., and Robins, J.M., Arch. Environ. Health 39, (1984),431.

16) R.K. Mehra and D.R. Winge, J. Cell Biochem. 45, (1991), 30.

17) I. Macreadie, L. Castelli, R. Mehra, J. Thorvaldsen and D. Winge, Biotechnol. Appl. Biochem. 19, (1994), 265.

18) K.B. Feingold, M. Moa-Qiang, T. Mauro, R. Warren, P.M. Elias and Bench, G., Submitted to British Journal of Dermatology.

19) T. Mauro, U. Rassner, G. Bench, K.R. Feingold, P. Elias, C. Cullander, Journal of Investigative Medicine, 44 (1), (1996), 186A

20) D.H. Morse, G.S. Bench, A.E. Pontau, S.P.H.T. Freeman, D.W. Heikkinen and P.R. Bach, Nucl. Instr. and Meth. B99, (1995), 427. 


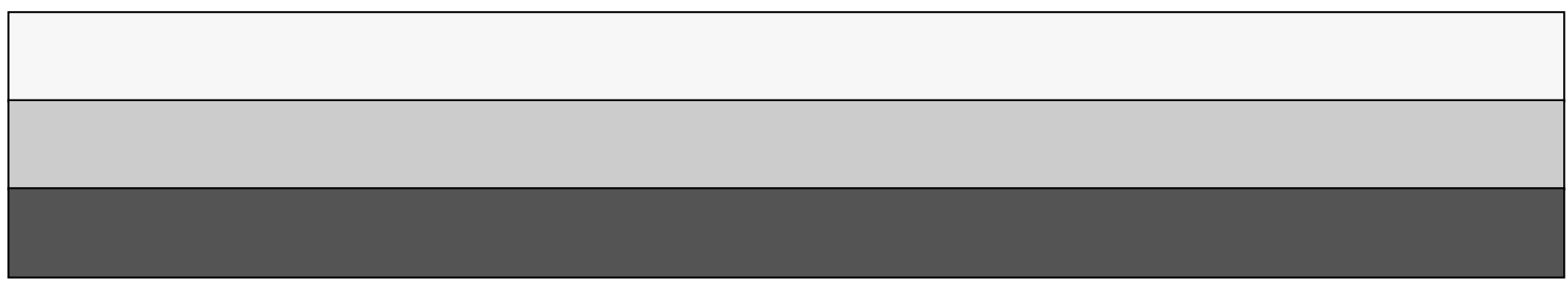

